\title{
Free fatty acid processing diverges in human pathologic insulin resistance conditions
}

\author{
Hilal Sekizkardes, ${ }^{1}$ Stephanie Therese Chung, ${ }^{2}$ Shaji Chacko, ${ }^{3}$ Morey W. Haymond, ${ }^{3}$ Megan Startzell, ${ }^{2}$ Mary Walter, ${ }^{2}$ \\ Peter J. Walter, ${ }^{2}$ Marissa Lightbourne, ${ }^{1}$ and Rebecca J. Brown ${ }^{2}$ \\ ${ }^{1}$ National Institute of Child Health and Human Development, ${ }^{2}$ National Institute of Diabetes and Digestive and Kidney Diseases, NIH, Bethesda, Maryland, USA. ${ }^{3}$ hildren's Nutrition Research Center, \\ Department of Pediatrics, US Department of Agriculture/Agricultural Research Service, Baylor College of Medicine, Houston, Texas, USA.
}

\begin{abstract}
BACKGROUND. Postreceptor insulin resistance (IR) is associated with hyperglycemia and hepatic steatosis. However, receptorlevel IR (e.g., insulin receptor pathogenic variants, INSR) causes hyperglycemia without steatosis. We examined 4 pathologic conditions of IR in humans to examine pathways controlling lipid metabolism and gluconeogenesis.
\end{abstract}

\begin{abstract}
METHODS. Cross-sectional study of severe receptor IR (INSR, $n=7$ ) versus postreceptor IR that was severe (lipodystrophy, $n=14$ ), moderate (type 2 diabetes, $n=9$ ), or mild (obesity, $n=8$ ). Lipolysis (glycerol turnover), hepatic glucose production (HCP), gluconeogenesis (deuterium incorporation from body water into glucose), hepatic triglyceride (magnetic resonance spectroscopy), and hepatic fat oxidation (plasma $\beta$-hydroxybutyrate) were measured.
\end{abstract}

\begin{abstract}
RESULTS. Lipolysis was 2- to 3-fold higher in INSR versus all other groups, and HCP was 2-fold higher in INSR and lipodystrophy versus type 2 diabetes and obesity $(P<0.001)$, suggesting severe adipose and hepatic IR. INSR subjects had a higher contribution of gluconeogenesis to HCP, approximately $77 \%$, versus $52 \%$ to $59 \%$ in other groups $(P=0.0001)$. Despite high lipolysis, INSR subjects had low hepatic triglycerides (0.5\% [interquartile range $0.1 \%-0.5 \%$ ]), in contrast to lipodystrophy (10.6\% [interquartile range $2.8 \%-17.1 \%$ ], $P<0.0001)$. $\beta$-hydroxybutyrate was 2 - to 7 -fold higher in INSR versus all other groups $(P<0.0001)$, consistent with higher hepatic fat oxidation.
\end{abstract}

CONCLUSION. These data support a key pathogenic role of adipose tissue IR to increase glycerol and FFA availability to the liver in both receptor and postreceptor IR. However, the fate of FFA diverges in these populations. In receptor-level IR, FFA oxidation drives gluconeogenesis rather than being reesterified to triglyceride. In contrast, in postreceptor IR, FFA contributes to both gluconeogenesis and hepatic steatosis.

TRIAL REGISTRATION. ClinicalTrials.gov NCT01778556, NCT00001987, and NCT02457897.

FUNDING. National Institute of Diabetes and Digestive and Kidney Diseases, US Department of Agriculture/Agricultural Research Service 58-3092-5-001.

\section{Introduction}

Insulin is a hormone that signals the fed state, promoting a switch from net glucose production to net glucose uptake and shifting lipids from lipid metabolism to lipid storage. In adipose tissue, insulin's major role is to suppress lipolysis, thus reducing plasma concentrations of free fatty acid (FFA) and glycerol availability. In the liver, insulin suppresses glucose production and increases de novo lipogenesis (DNL) in the presence of carbohydrate precursors (1). Brown and Goldstein first proposed that hepatic insulin resistance is selective in type 2 diabetes. In the selective insulin resistance model, the ability of insulin to suppress hepatic glucose production is impaired, contributing to hyperglycemia, where-

Conflict of interest: The authors have declared that no conflict of interest exists. Copyright: ( 2020 , American Society for Clinical Investigation.

Submitted: December 6, 2019; Accepted: March 17, 2020; Published: June 2, 2020

Reference information: / Clin Invest. 2020;130(7):3592-3602.

https://doi.org/10.1172/JCl135431. as the ability of insulin to stimulate DNL is intact, and in fact is upregulated by compensatory hyperinsulinemia, contributing to hypertriglyceridemia and hepatic steatosis $(2,3)$.

In contrast to type 2 diabetes, inhibition of insulin signaling at the level of the insulin receptor causes extreme insulin resistance in all pathways downstream of the insulin receptor. In humans, this phenomenon clinically manifests in patients with pathogenic variants of the insulin receptor (INSR) as a phenotype of hyperglycemia, with low hepatic and serum triglycerides, presumably from failure of insulin to stimulate DNL (4). However, although DNL contributes to excess hepatic and serum triglyceride in insulinresistant patients, it is not the major contributor (5). Instead, the major contributor appears to be reesterification of circulating FFAs, which are present in high abundance due to failure of insulin to suppress lipolysis. As hepatic reesterification of FFAs is not thought to be insulin dependent (6), the lack of hepatic steatosis in rodents and humans with receptor-level insulin signaling defects suggests either low FFA delivery to the liver due to low lipolysis, or 


\section{Table 1. Baseline characteristics}

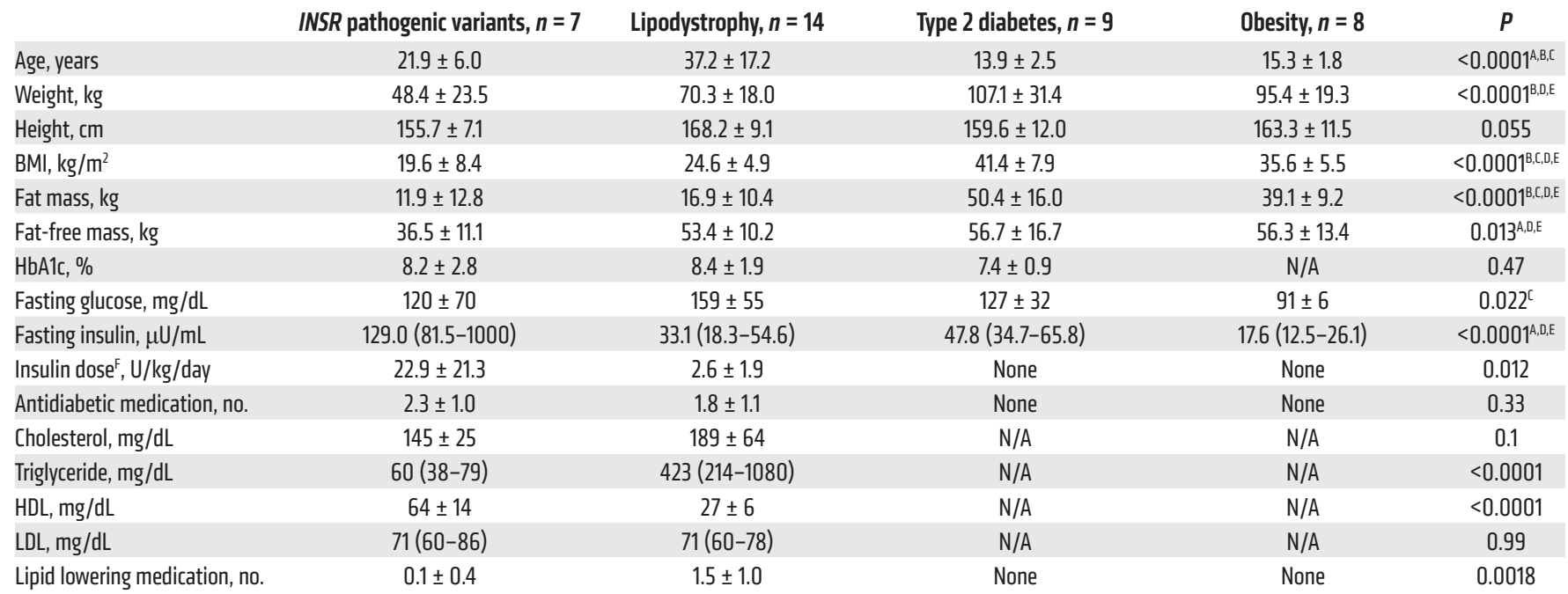

Data are mean \pm SD or median (interquartile range) based on data distribution. ${ }^{A} P<0.05$ for INSR pathogenic variants versus lipodystrophy. ${ }^{\mathrm{B} P}<0.05$ for lipodystrophy versus type 2 diabetes. ${ }^{C} P<0.05$ for lipodystrophy versus obesity. ${ }^{D} P<0.05$ for INSR pathogenic variants versus type 2 diabetes. ${ }^{E} P<0.05$ for INSR pathogenic variants obesity. ${ }^{\mathrm{F}} \mathrm{Among}$ insulin users only. N/A, not available.

shunting of FFAs in the liver into $\beta$-oxidation to drive gluconeogenesis, rather than reesterification.

The objectives of the current study were to define the role of lipolysis in contributing to hepatic glucose production versus hepatic lipid accumulation in states of receptor-level versus postreceptor insulin resistance in humans. We studied subjects with severe receptor-level insulin resistance due to INSR pathogenic variants, compared with subjects with severe postreceptor insulin resistance due to lipodystrophy. Additional cohorts with mild to moderate postreceptor insulin resistance (type 2 diabetes, obesity) were included for comparison. We hypothesized that both cohorts with severe insulin resistance (receptor-level and postreceptor) would have higher whole-body lipolysis compared with the less insulin-resistant groups (type 2 diabetes and obesity). However, we further hypothesized that the fate of FFAs in the liver would diverge in receptor-level versus postreceptor insulin resistance: in subjects with lipodystrophy, high FFA flux from adipose tissue lipolysis would thus contribute to both gluconeogenesis and hepatic steatosis. By contrast, in subjects with INSR pathogenic variants, FFAs would be shunted toward $\beta$-oxidation, resulting in increased ketone production and gluconeogenesis, with lack of hepatic steatosis.

\section{Results}

Baseline characteristics. Baseline characteristics are given in Table 1. Seven subjects with INSR pathogenic variants, 14 with lipodystrophy, 9 adolescents within 2 weeks of diagnosis of type 2 diabetes, and 8 obese nondiabetic adolescents were studied (Figure 1). Five of 7 subjects with insulin receptor dysfunction had biallelic pathogenic variants in INSR (resulting in more severe insulin resistance), whereas 2 had monoallelic variants (resulting in less severe insulin resistance). Four of 14 subjects with lipodystrophy had generalized lipodystrophy, while 10 had partial lipodystrophy. Genetic details of subjects with lipodystrophy and INSR pathogenic vari- ants are in Supplemental Table 1 (supplemental material available online with this article; https://doi.org/10.1172/JCI135431DS1).

Subjects with lipodystrophy were older than the other groups (mean \pm SD was 21.9 \pm 6.0 years for INSR pathogenic variants, 37.2 \pm 17.2 for lipodystrophy, $13.9 \pm 3.5$ for type 2 diabetes, and $15.3 \pm$ 1.8 for obesity, $P<0.0001)$; unadjusted comparisons are presented with age-adjusted analyses in Supplemental Table 2. Subjects with INSR pathogenic variants and lipodystrophy had lower BMI compared with subjects with type 2 diabetes and obesity (19.6 \pm $8.4 \mathrm{~kg} / \mathrm{m}^{2}, 24.6 \pm 4.9 \mathrm{~kg} / \mathrm{m}^{2}, 41.4 \pm 7.9 \mathrm{~kg} / \mathrm{m}^{2}, 35.6 \pm 5.5 \mathrm{~kg} / \mathrm{m}^{2}$, $P<0.0001)$. Likewise, fat mass was lower in subjects with INSR pathogenic variants and lipodystrophy compared with those with type 2 diabetes and obesity $(11.9 \pm 12.8 \mathrm{~kg}, 16.9 \pm 10.4 \mathrm{~kg}, 50.4 \pm$ $16.0 \mathrm{~kg}$, and $39.1 \pm 9.2 \mathrm{~kg}$, respectively, $P<0.0001)$.

The 3 groups with diabetes (INSR pathogenic variants, lipodystrophy, and type 2 diabetes) did not have statistically different glycemia control as measured by HbA1c (mean range 7.4\%-8.4\%, $P=$ 0.47). Fasting plasma glucose was higher in subjects with lipodystrophy compared with those with obesity but was otherwise comparable among groups. Fasting insulin was higher in subjects with INSR pathogenic variants compared with all other groups. Four of 7 (57\%) subjects with INSR pathogenic variants and 9 of 14 (64\%) with lipodystrophy were taking insulin at the time of the study. Insulin doses among insulin users were approximately 10-fold higher in subjects with INSR pathogenic variants compared with subjects with lipodystrophy, again consistent with greater insulin resistance in the INSR group $(22.9 \pm 21.3 \mathrm{U} / \mathrm{kg} /$ day vs. $2.6 \pm 1.9 \mathrm{U} / \mathrm{kg} /$ day, $P=$ 0.012). The number of antidiabetic medications and use of metformin was similar in subjects with INSR pathogenic variants and lipodystrophy. However, 5 of 7 subjects with INSR pathogenic variants were taking metreleptin as an experimental drug for diabetes versus none of the subjects with lipodystrophy. Subjects with type 2 diabetes and obesity were not taking any medications. 
INSR
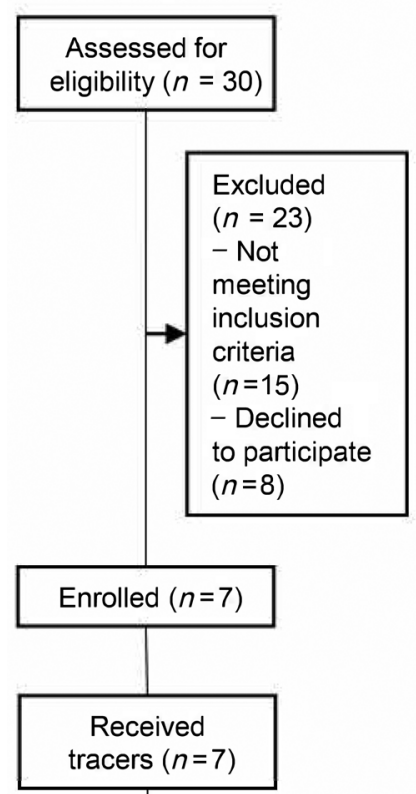

Analyzed $(n=7)$
Lipodystrophy

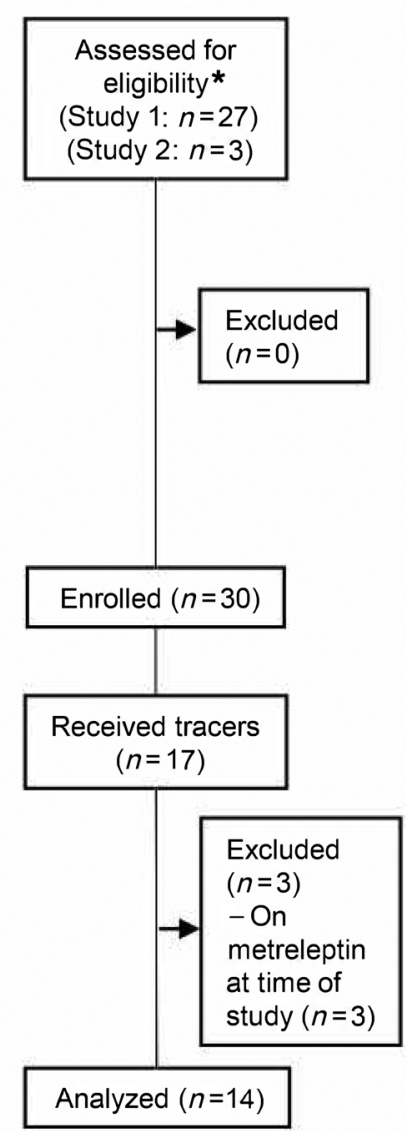

Type 2 Diabetes

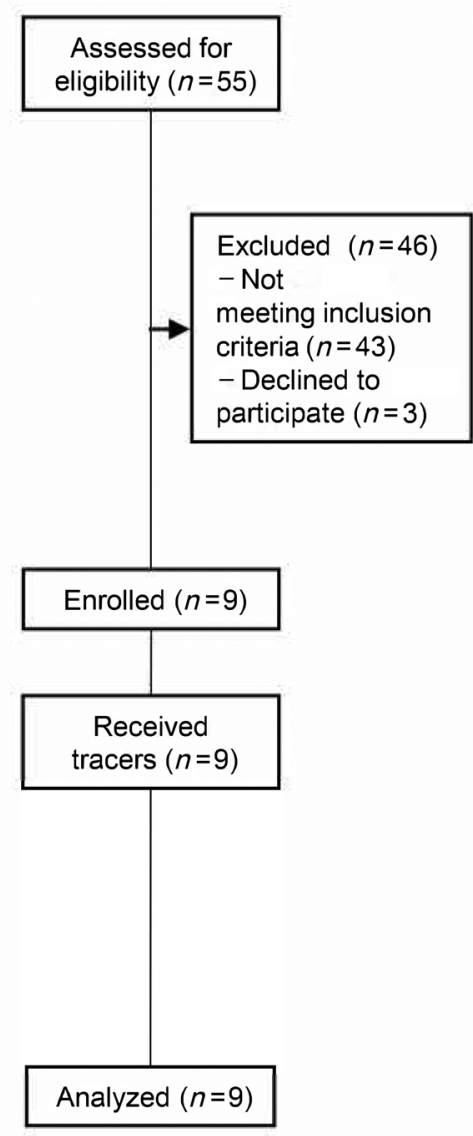

Obesity

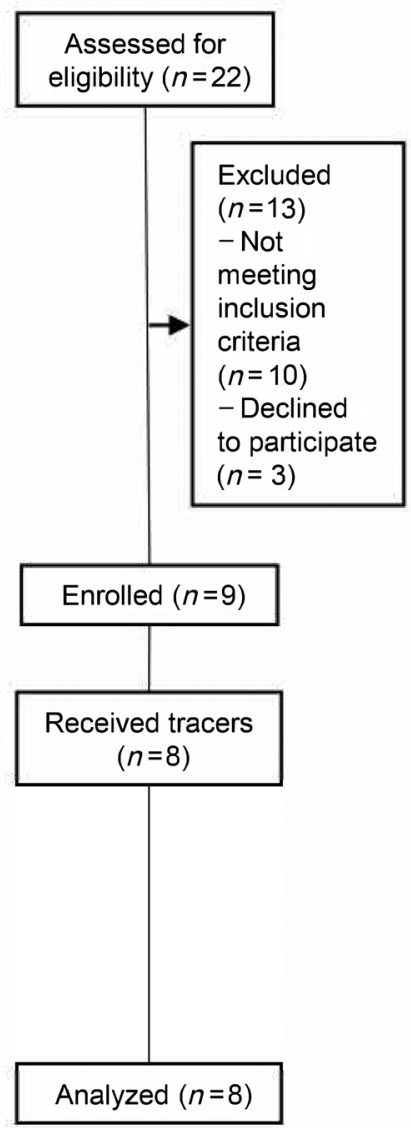

Figure 1. CONSORT flow diagram. Subjects with pathogenic variants of the insulin receptor (INSR), lipodystrophy, type 2 diabetes, and obesity were recruited in the context of multiple clinical trials. Subjects who received stable isotope tracers for measurement of lipolysis, hepatic glucose production, and gluconeogenesis were included in this cross-sectional study. *ClinicalTrials.gov identifiers NCT01778556 (study 1) and NCT00001987 (study 2).

Lipolysis was elevated in subjects with severe receptor-level insulin resistance (INSR pathogenic variants). Whole-body lipolysis was measured as glycerol rate of appearance by infusion of ${ }^{2} \mathrm{H}_{5}$-glycerol and normalized to body weight. Lipolysis was elevated in subjects with INSR pathogenic variants compared with all groups with postreceptor insulin resistance: approximately 2 -fold higher than subjects with lipodystrophy and type 2 diabetes, and approximately 3 -fold higher than subjects with obesity $(P=0.0001)$ (Figure $2 \mathrm{~A}$ ), consistent with failure of insulin to suppress lipolysis in the context of severe insulin resistance. The results were unchanged when glycerol turnover was normalized to fat mass as a measure of adipose tissue lipolysis; however, patients with generalized lipodystrophy were outliers in this analysis due to low fat mass (Supplemental Table 2 and Supplemental Figure 1). Similarly, plasma glycerol concentrations were approximately 2 -fold higher in subjects with INSR pathogenic variants compared with subjects with obesity $(P=0.013)$ and approximately $40 \%$ and $80 \%$ higher compared with subjects with type 2 diabetes and lipodystrophy, although the latter differences were not statistically significant (Figure 2B).

In subjects with INSR pathogenic variants and lipodystrophy, FFA turnover was measured as palmitate rate of appearance by stable-isotope infusion of $\left[\mathrm{U}-{ }^{13} \mathrm{C}_{16}\right]$ palmitate normalized to body weight. There was no difference in palmitate turnover between the 2 groups (Figure 2C). Palmitate turnover was not measured in subjects with type 2 diabetes or obesity. Plasma palmitate concentrations were comparable in subjects with INSR pathogenic variants and lipodystrophy and were higher in subjects with type 2 diabetes compared with those with obesity (Figure 2D). Similarly, plasma total FFA concentrations were comparable in subjects with INSR pathogenic variants and lipodystrophy and were higher in subjects with INSR pathogenic variants and type 2 diabetes compared with those with obesity, and higher in subjects with type 2 diabetes compared with lipodystrophy (Figure 2E).

The contribution of gluconeogenesis to hepatic glucose production was higher in receptor-level versus postreceptor insulin resistance. Hepatic glucose production rates were calculated from glucose appearance rate measurements under fasting steady-state conditions after the infusion of $\left[6,6-{ }^{2} \mathrm{H}_{2}\right]$ glucose and were normalized to body weight. The fraction of glucose derived from gluconeogenesis was measured using incorporation of deuterium on newly formed glucose from body water enriched with ${ }^{2} \mathrm{H}_{2} \mathrm{O}$. Absolute rates of gluconeogenesis and glycogenolysis were calculated from glucose appearance and fractional gluconeogenesis measurements. Rates of hepatic glucose production were approximately 2-fold higher in subjects with severe insulin resistance due to both INSR pathogenic variants and lipodystrophy compared with 
A

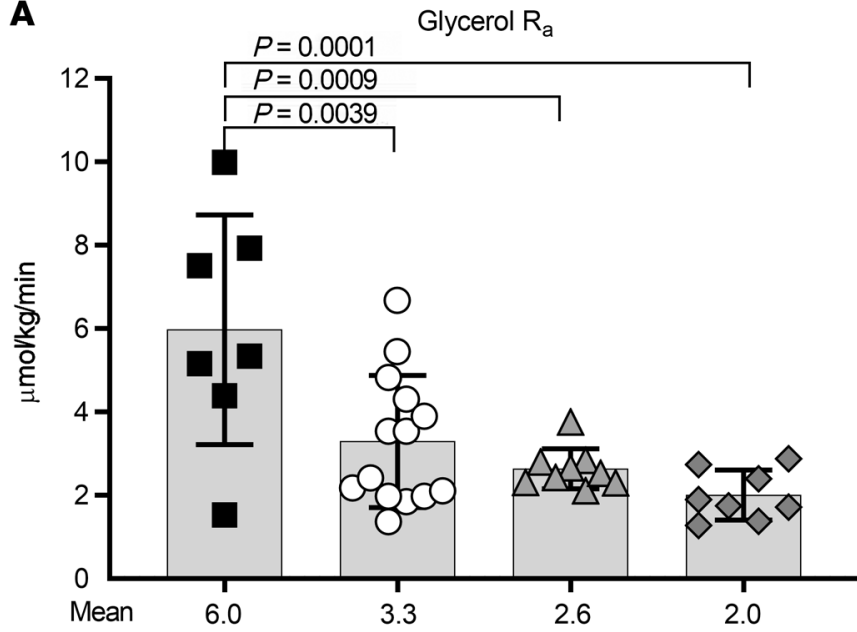

C

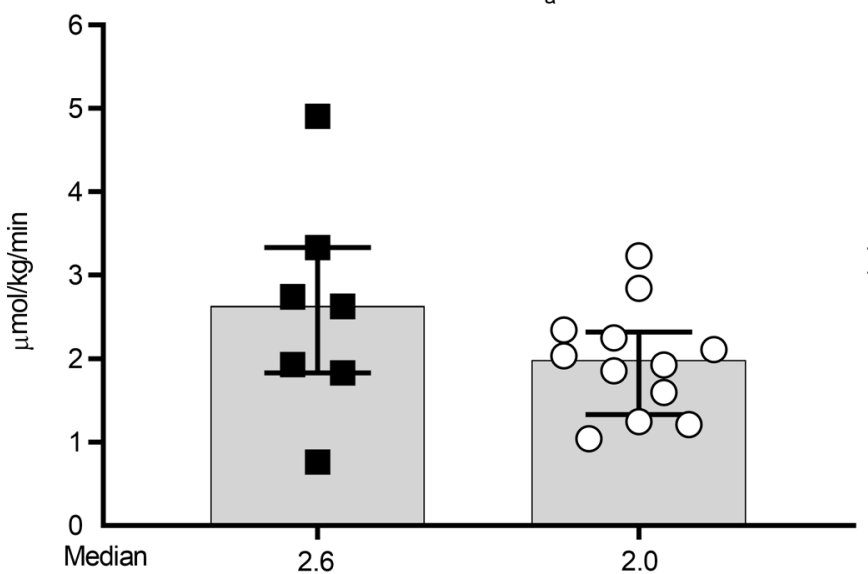

E

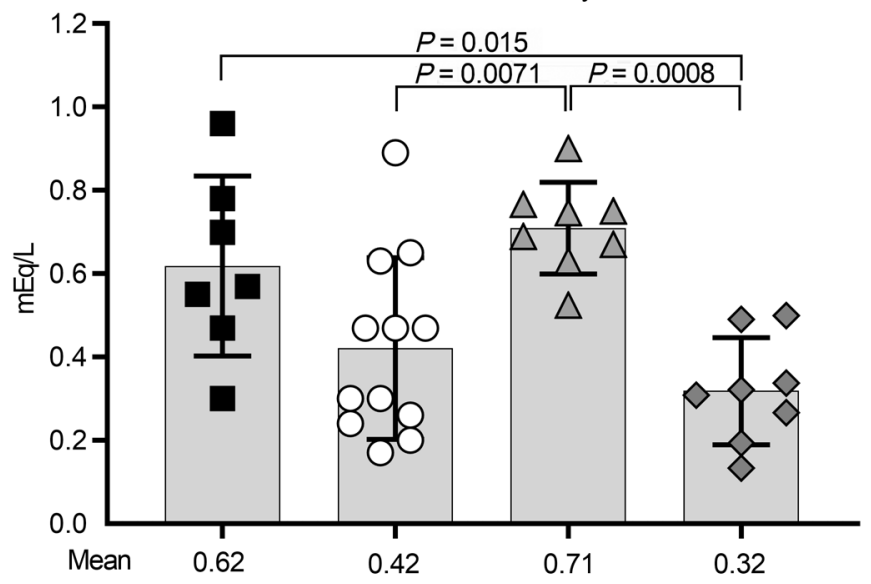

B

Plasma Glycerol

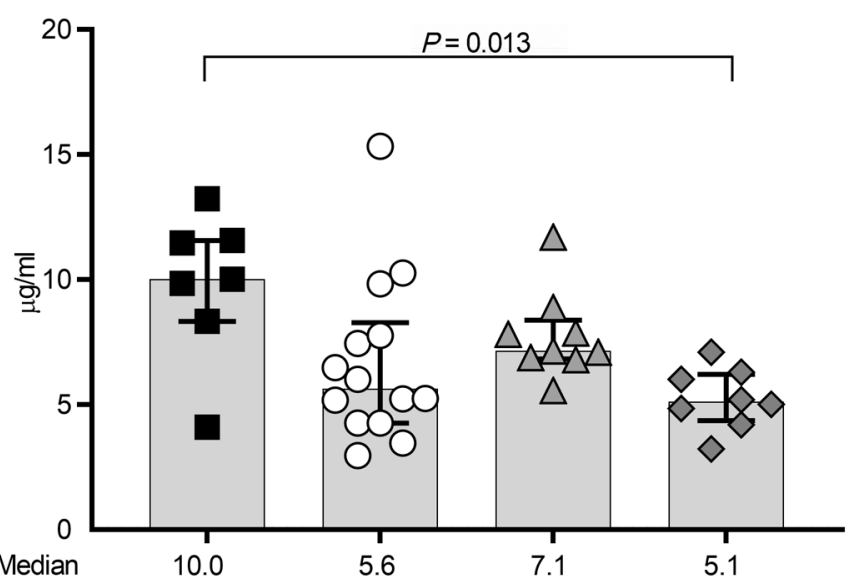

D

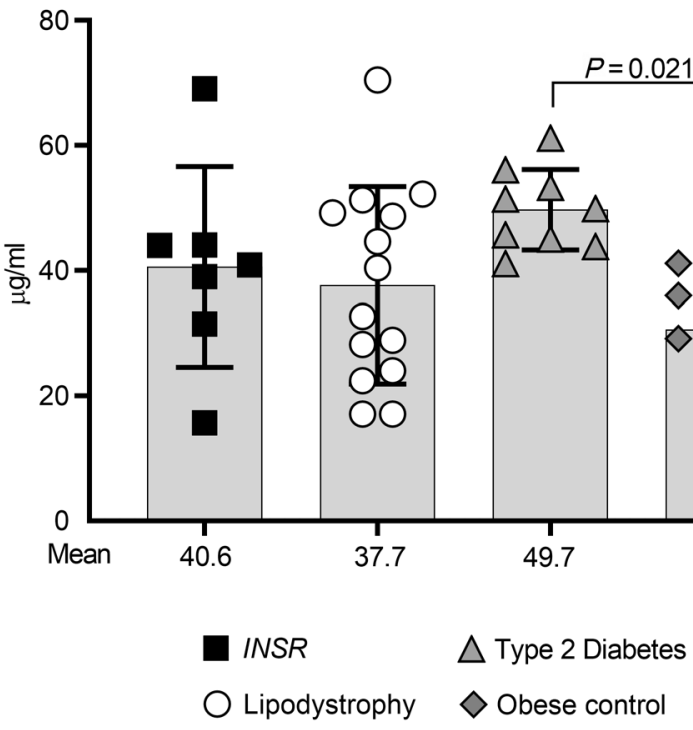

Figure 2. Measures of lipid metabolism. Lipolysis (A), measured as glycerol rate of appearance $\left(R_{2}\right)$ per kilogram of body weight, was highest in subjects with pathogenic variants of the insulin receptor (INSR, black squares) compared with those with lipodystrophy (white circles), type 2 diabetes (gray triangles), or obesity (gray diamonds). Similarly, plasma glycerol concentration (B) was highest in subjects with INSR pathogenic variants, although it was only significantly different from subjects with obesity. FFA turnover, measured as palmitate $\mathrm{R}_{\mathrm{a}}$ per kilogram of body weight (C), plasma palmitate concentrations (D), and total FFA concentrations (E) were comparable in subjects with INSR pathogenic variants and lipodystrophy. Subjects with type 2 diabetes had higher palmitate (D) and total FFA (E) compared with those with obesity, and higher total FFA compared with those with lipodystrophy. Statistical comparisons were performed using mixed models with post hoc pairwise comparisons and Tukey's correction for multiplicity. subjects with type 2 diabetes and obesity (Figure 3A, $P<0.0001$ ). However, subjects with INSR pathogenic variants had a much higher fractional contribution of gluconeogenesis to hepatic glucose production at approximately $77 \%$, compared with only $52 \%$ to $59 \%$ in the other 3 groups (Figure 3B, $P=0.0001$ ). Similarly, absolute gluconeogenesis rates were highest in subjects with INSR pathogenic variants: $47 \%$ higher than lipodystrophy, and approximately 2 -fold higher than type 2 diabetes and obesity (Figure 3C,
$P<0.0001)$. The absolute glycogenolysis rate in subjects with lipodystrophy was 2-fold higher than those with INSR pathogenic variants, type 2 diabetes, and obesity (Figure 3D, $P<0.0001$ ).

The concentration of plasma lactate, a principle substrate for gluconeogenesis, was approximately $50 \%$ higher in subjects with lipodystrophy and type 2 diabetes compared with those with INSR pathogenic variants and obesity $(P=0.0067$, Figure $3 \mathrm{E})$. The concentration of plasma alanine, another substrate for gluco- 
neogenesis, was higher in subjects with INSR pathogenic variants compared with those with obesity $(P=0.011)$ but was otherwise comparable across groups (Figure $3 \mathrm{~F}$ ).

Hepatic triglyceride content and fasting plasma triglycerides were low in severe receptor-level insulin resistance (INSR pathogenic variants) and high in postreceptor insulin resistance (lipodystrophy). Hepatic triglyceride content, measured by proton magnetic resonance spectroscopy, was significantly higher in subjects with lipodystrophy $(n=14)$ compared with subjects with INSR pathogenic variants $(n=3)$ (10.6\% [interquartile range $2.8 \%-17.1 \%$ ] vs. $0.5 \%$ [interquartile range $0.1 \%-0.5 \%$ ], $P<0.0001$, Figure $4 \mathrm{~A}$ ), consistent with presence of nonalcoholic fatty liver disease (NAFLD) in subjects with lipodystrophy, and absence of this condition in those with INSR pathogenic variants. Hepatic triglyceride content was not measured in subjects with type 2 diabetes or obesity.

Fasting plasma triglycerides were normal in subjects with INSR pathogenic variants and markedly elevated in subjects with lipodystrophy $(60 \mathrm{mg} / \mathrm{dL}$ [interquartile range 38-79 mg/dL] vs. 423 $\mathrm{mg} / \mathrm{dL}$ [interquartile range $214-1080 \mathrm{mg} / \mathrm{dL}$ ],$P<0.0001$, Figure 4B). Similarly, HDL was normal in subjects with INSR pathogenic variants and markedly low in subjects with lipodystrophy $(64 \pm$ 14 vs. $27 \pm 6 \mathrm{mg} / \mathrm{dL}, P<0.0001)$. Total cholesterol $(145 \pm 25 \mathrm{mg} /$ dL vs. $189 \pm 64 \mathrm{mg} / \mathrm{dL}, P=0.10)$ and LDL-cholesterol $(71 \mathrm{mg} / \mathrm{dL}$ [interquartile range $60-86 \mathrm{mg} / \mathrm{dL}$ ] vs. $71 \mathrm{mg} / \mathrm{dL}$ [interquartile range $60-78 \mathrm{mg} / \mathrm{dL}], P=0.99$ ) were comparable in subjects with INSR pathogenic variants and lipodystrophy, respectively. Lipids were not available in subjects with type 2 diabetes and obesity.

Hepatic fatty acid oxidation was higher in receptor-level versus postreceptor insulin resistance. $\beta$-Hydroxybutyrate, measured by enzymatic assay, was assessed as a surrogate measure of hepatic fat oxidation. $\beta$-Hydroxybutyrate was 4 - to 10 -fold higher in subjects with INSR pathogenic variants compared with all other groups $(0.73 \mathrm{mmol} / \mathrm{L}$ [interquartile range $0.44-1.26 \mathrm{mmol} / \mathrm{L}$ ] in INSR vs. $0.35 \mathrm{mmol} / \mathrm{L}$ [interquartile range $0.28-0.43 \mathrm{mmol} / \mathrm{L}$ ] in lipodystrophy, $0.10 \mathrm{mmol} / \mathrm{L}$ [interquartile range $0.08-0.11$ $\mathrm{mmol} / \mathrm{L}$ ] in type 2 diabetes, and $0.11 \mathrm{mmol} / \mathrm{L}$ [interquartile range $0.10-0.13 \mathrm{mmol} / \mathrm{L}]$ in obesity, $P<0.0001)$ consistent with higher hepatic fat oxidation in the INSR group (Figure 4C). Subjects with generalized lipodystrophy were not outliers in this analysis (Supplemental Figure 3 and Supplemental Table 2).

Lipolysis appears to drive gluconeogenesis, particularly in subjects with severe receptor-level insulin resistance (INSR pathogenic variants). In the combined patient population (INSR pathogenic variants, lipodystrophy, type 2 diabetes, and obesity), there were significant correlations between lipolysis (glycerol rate of appearance per kg), $\beta$-hydroxybutyrate, gluconeogenesis, and hepatic glucose production (Table 2). In the combined cohorts of INSR pathogenic variants and lipodystrophy in whom palmitate flux was measured, palmitate rate of appearance per kilogram was also a significant predictor of $\beta$-hydroxybutyrate, gluconeogenesis, and hepatic glucose production. Among the separate subgroups, these correlations were strongest in subjects with INSR pathogenic variants (e.g., $R^{2} 0.56-0.73$ for correlations of lipolysis and palmitate rate of appearance with gluconeogenesis and hepatic glucose production). These findings are consistent with a model in which adipose tissue insulin resistance results in high lipolysis, leading to increased hepatic delivery of glycerol and FFAs. $\beta$-Oxidation of FFAs both increases $\beta$-hydroxybutyrate and drives gluconeogenesis.

\section{Discussion}

In this study, we investigated mechanisms by which insulin regulates hepatic glucose and triglyceride production in humans with severe receptor-level insulin resistance (pathogenic variants of the insulin receptor) compared with severe, moderate, and mild postreceptor insulin resistance (lipodystrophy, type 2 diabetes, and obesity). We observed elevated lipolysis rates in subjects with INSR pathogenic variants, with comparable FFA flux and total FFA levels in subjects with INSR pathogenic variants and lipodystrophy, consistent with severe insulin resistance. We also observed higher hepatic glucose production, consistent with higher hepatic insulin resistance, in subjects with both INSR pathogenic variants and lipodystrophy compared with subjects with type 2 diabetes and obesity. However, subjects with INSR pathogenic variants had a markedly higher contribution of gluconeogenesis to hepatic glucose production. The high gluconeogenesis rate in subjects with INSR pathogenic variants appeared to be driven by high lipolytic rates, leading to increased glycerol availability as a substrate for gluconeogenesis. Despite high glycerol and FFA availability due to high lipolysis, subjects with INSR pathogenic variants had low hepatic and circulating triglycerides. The coexistence of increased lipolysis, high hepatic gluconeogenesis, and low hepatic triglycerides in subjects with INSR pathogenic variants suggests that glycerol and FFA from lipolysis drive hepatic gluconeogenesis and not hepatic lipogenesis in these subjects with severe receptor-level insulin resistance.

These data provide direct kinetic evidence in humans to support nonselective insulin resistance in this population. By contrast, our data in subjects with severe postreceptor insulin resistance due to lipodystrophy suggest that glycerol and FFA from lipolysis stimulates both hepatic gluconeogenesis and hepatic triglyceride accumulation. These clinical data provide further evidence to support the concept of selective hepatic insulin resistance in subjects with lipodystrophy proposed by Brown and Goldstein (2), such that insulin's action to suppress hepatic glucose production is impaired, whereas insulin's action to stimulate hepatic lipogenesis remains intact, and is stimulated by the presence of hyperinsulinemia.

Insulin and regulation of hepatic glucose production. Insulin has direct effects on the hepatocytes to suppress gluconeogenesis, largely mediated by FOXO transcription factors and cyclic AMP response element-binding protein (CREB) transcriptional complex (7-9). However, multiple rodent studies support the concept that insulin's action to suppress gluconeogenesis is largely indirect and is mediated by suppression of adipose tissue lipolysis (10-12). Suppression of lipolysis reduces glycerol availability, which is a direct substrate for gluconeogenesis. Suppression of lipolysis also reduces FFA availability. FFA is oxidized in the hepatocyte to mitochondrial acetyl coA, which allosterically activates pyruvate carboxylase, thus increasing gluconeogenesis (1). Our data in humans with INSR pathogenic variants provide strong support for this model. In these subjects, higher lipolysis rates were observed compared with subjects with obesity, type 2 diabetes, or lipodystrophy, presumably due to the severe impairment in insulin signaling in adipose tissue. The resultant increase in glycerol and FFA availability is presumably driving the elevated rates of gluconeogenesis observed in these subjects, as insulin cannot directly suppress gluconeogenesis in the hepatocytes due to the mutation of the 
A

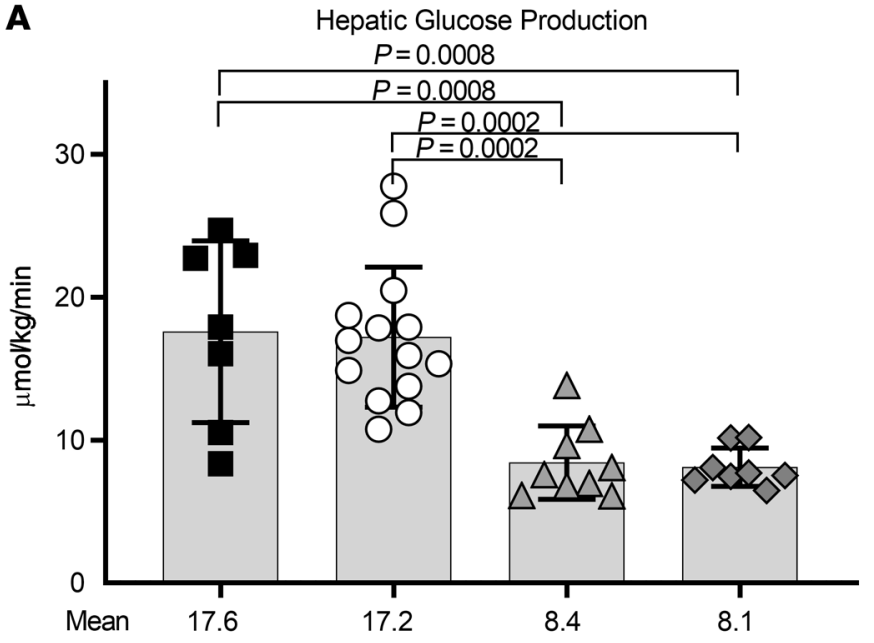

C

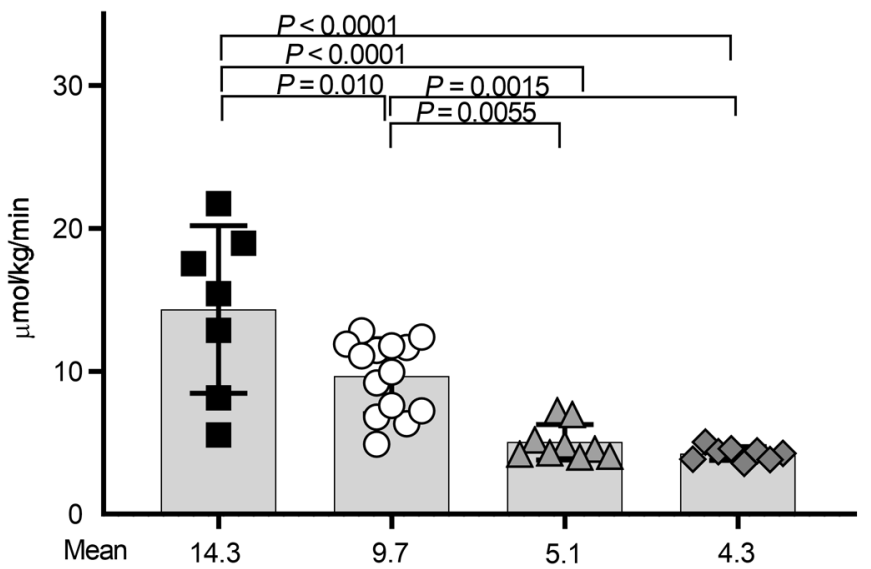

$\mathbf{E}$

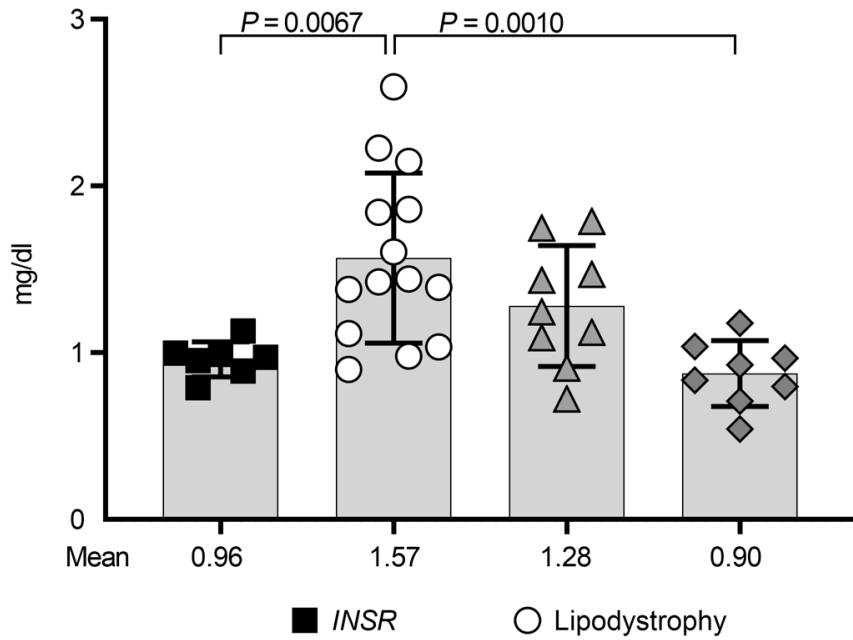

B

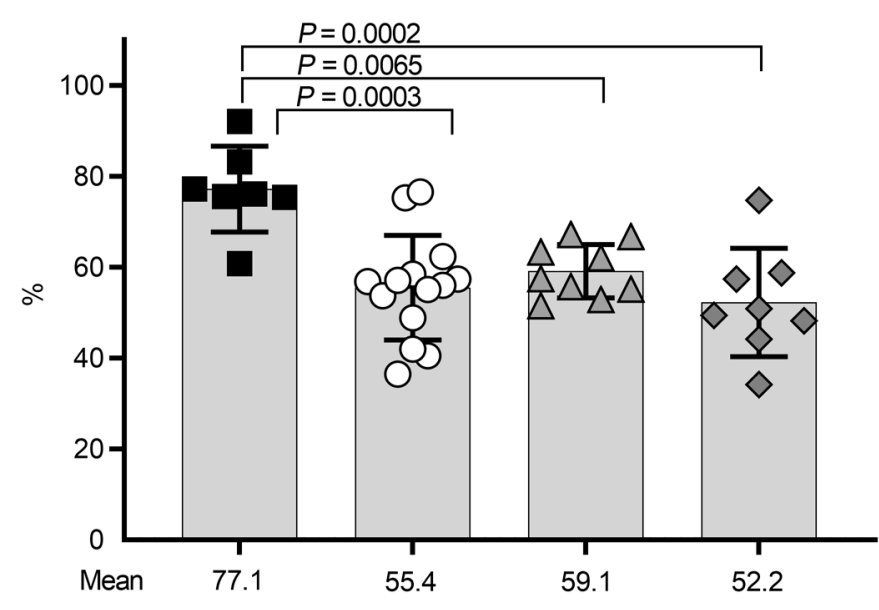

D

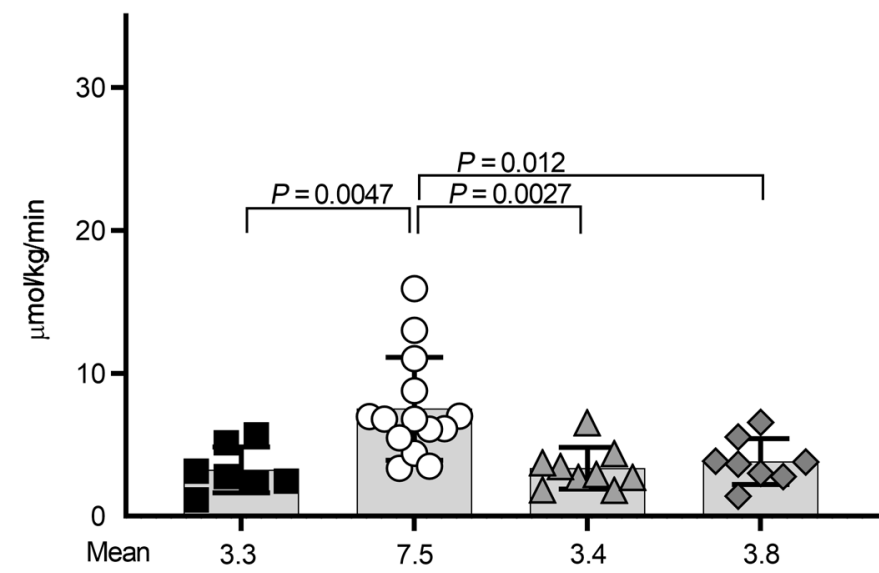

F

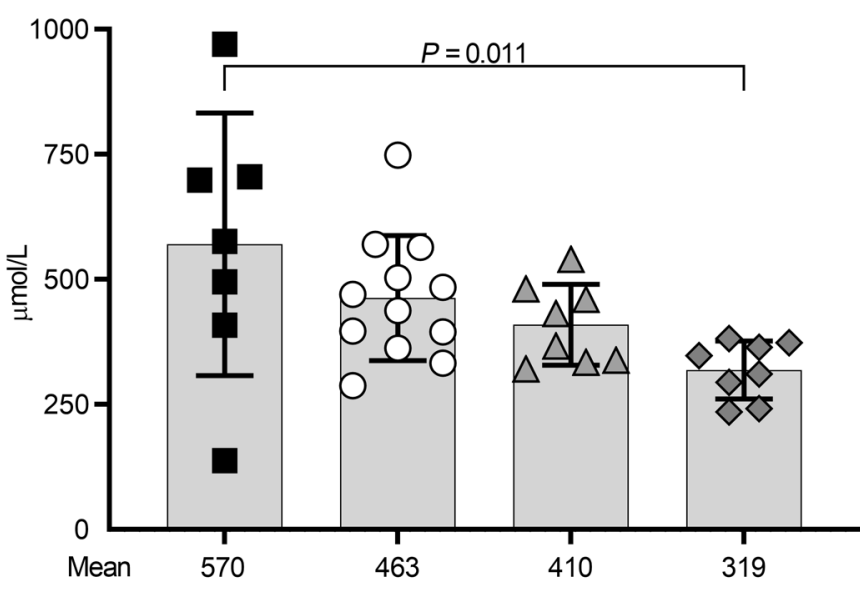

$\triangle$ Type 2 Diabetes
Fractional Gluconeogenesis

Absolute Glycogenolysis

Plasma Alanine

Obese control

Figure 3. Measures of glucose metabolism. Hepatic glucose production (A), measured as glucose rate of appearance per kilogram of body weight, was higher in subjects with pathogenic variants of the insulin receptor (INSR, black squares) and lipodystrophy (white circles) compared with those with type 2 diabetes (gray triangles) and obesity (gray diamonds). The percentage of hepatic glucose derived from gluconeogenesis (fractional gluconeogenesis), as well as absolute rates of gluconeogenesis and glycogenolysis, were measured using incorporation of deuterium into newly formed glucose after labeling of body water with ${ }^{2} \mathrm{H}_{2} \mathrm{O}$. Fractional gluconeogenesis (B) was higher in subjects with INSR pathogenic variants compared with all other groups. Accordingly, the absolute rate of gluconeogenesis (C) was highest in subjects with INSR pathogenic variants. The absolute rate of glycogenolysis (D) was highest in subjects with lipodystrophy. Plasma lactate (E), a substrate for gluconeogenesis, was highest in subjects with lipodystrophy, whereas plasma alanine (F), another gluconeogenic substrate, was highest in subjects with INSR pathogenic variants. Statistical comparisons were performed using mixed models with post hoc pairwise comparisons and Tukey's correction for multiplicity. 
A

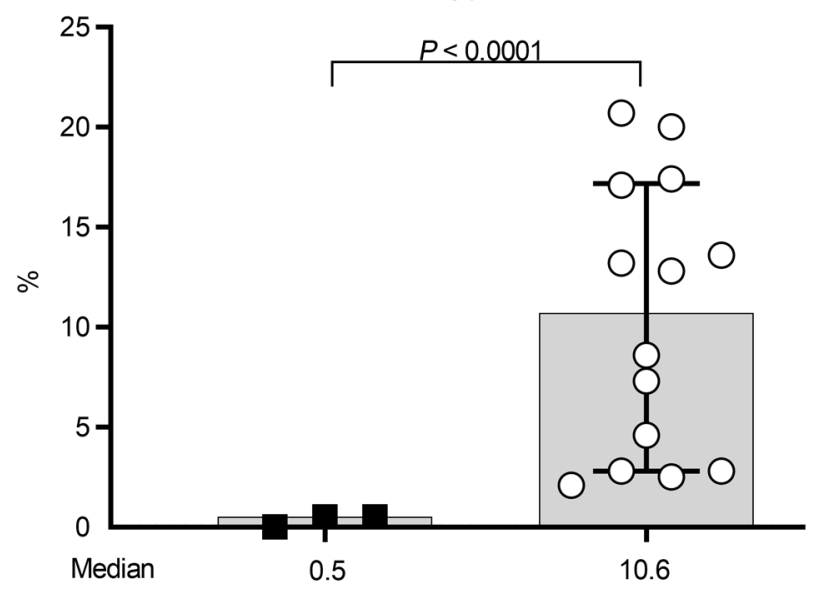

C

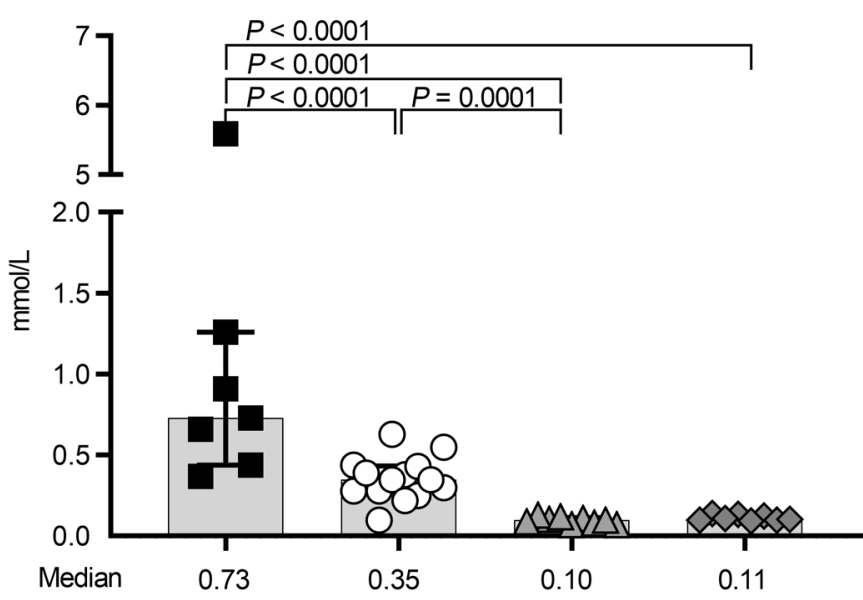

Plasma $\beta$-hydroxybutyrate
B

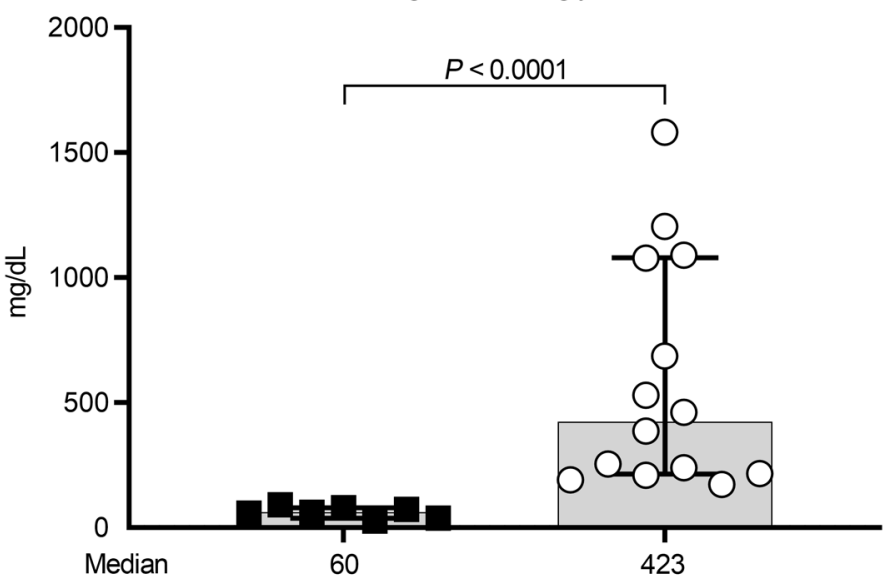

Figure 4. Measure of hepatic and plasma fat, and hepatic fatty acid oxidation. Hepatic triglyceride content (A), measured using magnetic resonance spectroscopy, and plasma triglycerides (B) were normal in subjects with pathogenic variants of the insulin receptor (INSR, black squares), and elevated, consistent with nonalcoholic fatty liver disease and severe dyslipidemia, in subjects with lipodystrophy (white circles). Plasma $\beta$-hydroxybutyrate (C), a measure of hepatic FFA oxidation, was higher in subjects with INSR pathogenic variants compared with all other groups, including lipodystrophy, type 2 diabetes (gray triangles), and obesity (gray diamonds). Statistical comparisons were performed using mixed models with post hoc pairwise comparisons and Tukey's correction for multiplicity.

insulin receptor. Plasma lactate was lower in subjects with INSR pathogenic variants compared with those with severe postreceptor insulin resistance due to lipodystrophy. This may be caused by increased lactate utilization as a substrate for gluconeogenesis; however, lactate flux measurements were not available to support this hypothesis. Although absolute gluconeogenesis was elevated in subjects with INSR pathogenic variants compared with all other groups, absolute rates of glycogenolysis did not differ from subjects with type 2 diabetes or obesity. This suggests that high gluconeogenesis is the major driver of high hepatic glucose production in subjects with INSR pathogenic variants. However, one may still consider that glycogenolysis is low in subjects with INSR pathogenic variants relative to their degree of hyperinsulinemia. Lower glycogenolysis rates in subjects with INSR pathogenic variants may be due to failure of insulin to generate the expected amount of glycogen in the postprandial state. Unfortunately, postprandial liver imaging for measurement of hepatic glycogen content was not available in this study to test this hypothesis.
In this study, we explored the mechanisms that may lead to increased hepatic triglyceride in insulin-resistant states. Consistent with prior observations, our subjects with postreceptor insulin resistance due to lipodystrophy had markedly elevated hepatic triglyceride (13-16), whereas subjects with receptor-level insulin resistance due to INSR pathogenic variants did not develop hepatic steatosis despite extreme insulin resistance and hyperinsulinemia $(4,16,17)$. Hepatic triglyceride accumulation is mainly derived from 3 sources: hepatic de novo lipogenesis from carbohydrate precursors, FFAs released from adipose tissue lipolysis, and dietary fatty acids (18). In subjects with obesity-associated NAFLD and lipodystrophy, the rate of de novo lipogenesis is elevated and contributes to hepatic triglyceride accumulation $(17,19,20)$. Insulin has direct effects to upregulate transcription of several genes promoting de novo lipogenesis (SREBP-1c, FAS, ACC, SCD1, lipin 1), and activates enzymes involving lipogenic pathways $(1,21)$. Overnutrition can stimulate de novo lipogenesis by increasing carbon precursors, by increasing insulin stimulation of lipogenic pathways, or both. A study from the 


\section{Table 2. Correlations among metabolic variables}

\begin{tabular}{|c|c|c|c|c|c|}
\hline \multirow[b]{2}{*}{ Predictor } & \multirow[b]{2}{*}{ Cohort(s) } & \multirow[b]{2}{*}{$r / P$} & \multicolumn{3}{|c|}{ Response variable } \\
\hline & & & $\begin{array}{l}\beta \text {-Hydroxybutyrate } \\
\text { (mmol/L) }\end{array}$ & $\begin{array}{l}\text { Cluconeogenesis } \\
(\mu \mathrm{mol} / \mathrm{kg} / \mathrm{min})\end{array}$ & $\begin{array}{l}\text { Hepatic glucose production } \\
(\mu \mathrm{mol} / \mathrm{kg} / \mathrm{min})\end{array}$ \\
\hline \multirow{10}{*}{$\begin{array}{l}\text { Clycerol } R_{a} \\
(\mu \mathrm{mol} / \mathrm{kg} / \mathrm{min})\end{array}$} & \multirow[t]{2}{*}{ All } & $r$ & 0.645 & 0.808 & 0.625 \\
\hline & & $P$ & $<0.0001$ & $<0.0001$ & $<0.0001$ \\
\hline & \multirow[t]{2}{*}{ INSR } & $r$ & 0.814 & 0.791 & 0.855 \\
\hline & & $P$ & 0.026 & 0.034 & 0.014 \\
\hline & \multirow[t]{2}{*}{ Lipodystrophy } & $r$ & -0.508 & 0.630 & 0.353 \\
\hline & & $P$ & 0.064 & 0.016 & 0.22 \\
\hline & \multirow[t]{2}{*}{ Type 2 diabetes } & $r$ & 0.516 & -0.473 & -0.355 \\
\hline & & $P$ & 0.16 & 0.20 & 0.35 \\
\hline & \multirow[t]{2}{*}{ Obesity } & $r$ & -0.146 & 0.050 & 0.126 \\
\hline & & $P$ & 0.73 & 0.91 & 0.77 \\
\hline \multirow{6}{*}{$\begin{array}{l}\text { Palmitate } R_{a} \\
(\mu \mathrm{mol} / \mathrm{kg} / \mathrm{min})\end{array}$} & \multirow[t]{2}{*}{ All } & $r$ & 0.502 & 0.715 & 0.607 \\
\hline & & $P$ & 0.029 & 0.001 & 0.006 \\
\hline & \multirow[t]{2}{*}{ INSR } & $r$ & 0.751 & 0.781 & 0.800 \\
\hline & & $P$ & 0.052 & 0.038 & 0.031 \\
\hline & \multirow[t]{2}{*}{ Lipodystrophy } & $r$ & -0.262 & 0.642 & 0.355 \\
\hline & & $P$ & 0.41 & 0.024 & 0.26 \\
\hline \multirow{10}{*}{$\begin{array}{l}\beta \text {-Hydroxybutyrate } \\
\text { (mmol/L) }\end{array}$} & \multirow{2}{*}{ All } & $r$ & & 0.764 & 0.643 \\
\hline & & $P$ & & $<0.0001$ & $<0.0001$ \\
\hline & \multirow[t]{2}{*}{ INSR } & $r$ & & 0.558 & 0.688 \\
\hline & & $P$ & & 0.19 & 0.088 \\
\hline & \multirow[t]{2}{*}{ Lipodystrophy } & $r$ & & -0.269 & -0.453 \\
\hline & & $P$ & & 0.35 & 0.10 \\
\hline & \multirow[t]{2}{*}{ Type 2 diabetes } & $r$ & & 0.030 & 0.191 \\
\hline & & $P$ & & 0.94 & 0.62 \\
\hline & \multirow[t]{2}{*}{ Obesity } & $r$ & & 0.071 & -0.373 \\
\hline & & $P$ & & 0.87 & 0.36 \\
\hline
\end{tabular}

INSR, pathogenic variants in the insulin receptor; $\mathrm{R}_{\mathrm{a}}$, rate of appearance. Bold type indicates statistically significant associations.

Biddinger lab showed that, in insulin-sensitive rodents, physiologic induction of lipogenic pathways in the liver (e.g., SREBP-1c transcription) was activated by feeding and was not dependent on insulin. However, in obesity-induced insulin resistance, the pathological induction of SREBP1c and other lipogenic genes that is needed to develop NAFLD or hypertriglyceridemia was insulin dependent (22). Furthermore, de novo lipogenesis rates are low in subjects with INSR pathogenic variants, comparable to healthy, insulin-sensitive controls (17). These observations are consistent with the selective insulin resistance model proposed by Brown and Goldstein, in which pathologic hyperinsulinemia drives hepatic de novo lipogenesis in obesity-associated, postreceptor insulin resistance.

Although increased de novo lipogenesis plays an important role in obesity-associated NAFLD, the dominant contributor to hepatic triglyceride accumulation was found to be hepatic reesterification of FFAs derived from adipose tissue lipolysis (5). In subjects with NAFLD, approximately $26 \%$ of hepatic and VLDL triglyceride are derived from de novo lipogenesis, whereas approximately $60 \%$ arose from circulating FFAs (presumably derived from adipose tissue) and 15\% from the diet. Thus, adipose tissue insulin resistance with the resultant increase in FFA availability appears to be the major mechanism leading to hepatic steatosis in humans with postreceptor insulin resistance.

Rodent studies suggest that hepatic FFA reesterification is insulin independent (6). If hepatic FFA reesterification in humans is independent of insulin receptor signaling, the high lipolytic rates with increased FFA availability in subjects with INSR pathogenic variants should lead to increased hepatic FFA reesterification and hepatic steatosis. Our observations of increased gluconeogenesis and low hepatic triglyceride in subjects with INSR pathogenic variants suggest that FFA delivered to the liver in these subjects is directed into mitochondrial $\beta$-oxidation, thus driving gluconeogenesis, rather than reesterified to triglyceride. Supporting this hypothesis, $\beta$-hydroxybutyrate, which is only generated by hepatic $\beta$-oxidation, was elevated in subjects with INSR pathogenic variants compared with all other groups. The molecular switch point routing cytosolic FFA into either $\beta$-oxidation or reesterification is movement of cytosolic FFA into mitochondria (18). Transport of FFA into the mitochondria is regulated by malonyl CoA, such that increased malonyl CoA inhibits FFA transport into mitochondria (23). In the fed state and during de novo lipogenesis, malonyl CoA levels rise, thus increasing the cytosolic FFA pool. Cytosolic FFA is then esterified with glycerol to form triglyceride (24). Thus, we speculate that, in subjects with INSR pathogenic variants, lack of insulin stimulation of de novo lipogenesis results in lower malonyl CoA. This would lead to increased FFA transport into mitochondria, increased $\beta$-oxidation of FFA, and stimulation of gluconeogenesis. An alternative explanation for low hepatic triglyceride in subjects with INSR pathogenic variants is increased hepatic triglyceride export in VLDL. Although VLDL secretion was not directly measured in this study, subjects with INSR pathogenic variants had low circulating triglycerides, which does not support increased VLDL secretion as a mechanism for low hepatic triglyceride content.

The strength of this study is the use of human models of severe insulin resistance at the receptor and postreceptor levels to characterize the fates of products of lipolysis in the liver. Due to the rarity of these subjects, prior studies have largely been limited to rodent models and mild forms of postreceptor insulin resistance in humans, such as obesity and NAFLD. Lipodystrophy and insulin receptor pathogenic variants are very rare conditions, and it is not possible to control for all variables in clinical studies. As a result, there was heterogeneity of etiology and severity among subjects with severe insulin resistance syndromes in this study, as well as baseline differences in age, 
body composition, and medication treatment among the patient cohorts. Sensitivity analyses adjusting for age differences among groups did not meaningfully alter the results (Supplemental Table 2). However, this approach has limited statistical validity due to the small overlap in ages between groups. While there were differences between cohorts in medication use (in particular, no medications in those with type 2 diabetes or obesity vs. multiple medications in those with INSR pathogenic variants and lipodystrophy), these medications are unlikely to account for the worse metabolic parameters seen in the latter groups (Supplemental Table 3). Instead, greater medication use in these groups is an attempt to control more severe metabolic dysregulation. In particular, use of metformin (which lowers hepatic glucose production) in all subjects with INSR pathogenic variants and $86 \%$ of subjects with lipodystrophy cannot account for the higher hepatic glucose production seen in these groups. Similarly, the higher use of lipid-lowering drugs in subjects with lipodystrophy cannot account for the higher circulating and hepatic triglycerides seen in this group. The differences in body composition, particularly fat mass, among groups requires careful interpretation. Glycerol $\mathrm{R}_{\mathrm{a}}$ is typically normalized to fat mass to provide a measure of adipose tissue lipolysis. However, because patients with generalized lipodystrophy have very low fat mass, most lipolysis in these patients is likely occurring in extra-adipose sites. Moreover, measurement of fat mass by dual-energy x-ray absorptiometry cannot distinguish between lipid in adipose tissue versus lipid in ectopic sites. We therefore normalized glycerol and palmitate $\mathrm{R}_{\mathrm{a}}$ to total body mass as measures of total body lipolysis and FFA turnover and performed sensitivity analyses normalizing these measures to fat mass and excluding patients with generalized lipodystrophy (Supplemental Table 2 and Supplemental Figure 1). All of these analyses showed higher lipolysis and FFA turnover in patients with INSR pathogenic variants, supporting the unique pathophysiology in this cohort. An additional limitation is that quantification of hepatic triglyceride content was available in only 3 subjects with INSR pathogenic variants, and in none of the subjects with type 2 diabetes or obesity. However, our finding of low hepatic triglyceride in the INSR pathogenic variant cohort is consistent with a prior publication (17). Finally, it would have been informative to measure de novo lipogenesis in this study; unfortunately, these measures were not available.

In conclusion, our data support a key pathogenic role of adipose tissue insulin resistance to increase glycerol and FFA availability to the liver in subjects with both postreceptor and receptor-level insulin resistance. However, the fate of FFA and glycerol diverges in these 2 populations. Our data support a model in which, in subjects with receptor-level insulin resistance, FFA is selectively directed toward $\beta$-oxidation, thus driving gluconeogenesis, rather than reesterified to hepatic triglyceride. By contrast, in our model, FFA derived from lipolysis in subjects with postreceptor insulin resistance both contributes to hepatic triglyceride accumulation and drives gluconeogenesis. Insulin is well established as a stimulator of de novo lipogenesis, and in subjects with postreceptor insulin resistance, hyperinsulinemia increases de novo lipogenesis per the selective insulin-resistance model of Brown and Goldstein (2). However, non-insulin-dependent reesterification of FFA derived from adipose tissue lipolysis has been shown to be the major mechanism contributing to hepatic steatosis in insulin-resistant states. Our data showing lack of hepatic triglyceride accumulation despite high FFA availability in subjects with receptor-level insulin resistance suggest that insulin is indirectly required for accumulation of hepatic triglyceride from hepatic FFA reesterification. We hypothesize that stimulation of de novo lipogenesis by hyperinsulinemia in postreceptor insulin resistance leads to increased hepatic malonyl CoA, thus directing FFAs away from $\beta$-oxidation and gluconeogenesis and toward reesterification. Further mechanistic studies are needed to test this hypothesis.

\section{Methods}

Subjects. Subjects with pathogenic variants of the insulin receptor (INSR), lipodystrophy, type 2 diabetes, and obesity were recruited in the context of multiple clinical trials. Subjects with lipodystrophy were recruited and studied between 2013 and 2016 (NCT01778556, NCT00001987), and those with INSR pathogenic variants during 2016 (NCT02457897) at the NIH. Subjects who received stable isotope tracers for measurement of lipolysis, hepatic glucose production, and gluconeogenesis were included in this cross-sectional study. Subjects with lipodystrophy included in this analysis were naive to metreleptin. Subjects continued their preadmission medications throughout the study, including oral hypoglycemic agents, lipid-lowering medications, and other medications either related or unrelated to lipodystrophy and its complications. Subjects with newly diagnosed type 2 diabetes and obese adolescent subjects without diabetes were enrolled in a study during 2012 at Texas Children's Hospital (25). Type 2 diabetes was diagnosed based on American Diabetes Association criteria (26). All subjects with type 2 diabetes were newly diagnosed and naive to insulin, oral hypoglycemic agents, and lipid-lowering medications. Subjects with obesity were matched for age and BMI, and diabetes was excluded using a $75 \mathrm{~g}$ oral glucose tolerance test using American Diabetes Association criteria.

Laboratory assays. In subjects with lipodystrophy and INSR pathogenic variants, glucose, insulin, triglyceride, total cholesterol, LDL, $\mathrm{HDL}, \mathrm{FFA}$, and HbA1c were measured using the standard techniques of the NIH Clinical Center after an 8- to 12-hour fast. In subjects with type 2 diabetes and obesity, insulin, FFA, and HbA1c (in subjects with diabetes) were measured as previously reported (25). Additional blood samples for measurement of palmitate, glycerol, lactate, alanine, and $\beta$-hydroxybutyrate were frozen at $-80^{\circ} \mathrm{C}$ before analysis; these assays were performed in the same laboratory for all subjects.

Plasma palmitate concentration was measured using ultra performance liquid chromatography tandem mass spectrometry (UPLCMS/MS) using a Thermo Fisher Scientific Vanquish UPLC with a Thermo Fisher Scientific Altis triple-quadrupole mass spectrometer with heated electrospray ionization (HESI-II) in negative ion mode. Quantitation of palmitate was based on mass fragmentation, mass selectivity, and retention time. Plasma glycerol concentration was measured using UPLC high-resolution accurate mass spectrometer (HRMS) using a Thermo Fisher Scientific Vanquish UPLC and a Thermo Fisher Scientific QExactive HRMS with HESI-II in negative ion mode $(2500 \mathrm{~V})$. Quantitation of glycerol was based on 91.0388 $\mathrm{m} / \mathrm{z}$ and the internal standard ${ }^{13} \mathrm{C}_{1}$-glycerol based on $92.0333 \mathrm{~m} / \mathrm{z}$ measured at 70,000 mass resolution. Plasma lactate concentration was measured using a Yellow Springs Instrument 2900 Biochemistry Analyzer. Plasma alanine concentration was measured via NMR 
spectroscopy using the 400-MHz proton Vantera Clinical Analyzer with LP4 deconvolution algorithm as previously described (27). $\beta$-Hydroxybutyrate was measured in plasma after a 10 - to 12 -hour fast using an enzymatic assay with colorimetric detection (Cayman Chemical). The intraassay and interassay coefficients of variation were $3.4 \%$ and $3.7 \%$, respectively.

Tracer dilution studies. Following a 10- to 12-hour fast, stable isotope tracers (Cambridge Isotope Laboratories) were used to measure glucose and glycerol turnover (all patient groups), and palmitate turnover (in subjects with lipodystrophy and INSR pathogenic variants) using the tracer dilution method. One catheter was inserted into the forearm vein to infuse stable isotopically labeled tracers. A second catheter was inserted into a vein in the contralateral hand or arm to obtain blood samples. A primed, continuous $\left[6,6-{ }^{2} \mathrm{H}_{2}\right] \mathrm{glu}-$ cose infusion was given for 3 hours in subjects with lipodystrophy and INSR pathogenic variants, and for 5 hours in subjects with type 2 diabetes and obesity. A primed, continuous ${ }^{2} \mathrm{H}_{5}$-glycerol infusion was given for 1 hour in subjects with lipodystrophy and INSR pathogenic variants, and for 5 hours in subjects with type 2 diabetes and obesity. In subjects with lipodystrophy and INSR pathogenic variants, an unprimed $\left[\mathrm{U}^{-13} \mathrm{C}_{16}\right]$ palmitate infusion was given for 1 hour. After these equilibration periods, blood samples to measure isotope enrichment were obtained every 10 minutes over a period of 30 minutes at steady state.

In subjects with lipodystrophy and INSR pathogenic variants, enrichments of $\left[6,6-{ }^{2} \mathrm{H}_{2}\right]$ glucose, ${ }^{2} \mathrm{H}_{5}$-glycerol, and $\left[\mathrm{U}^{-13} \mathrm{C}_{16}\right]$ palmitate were measured by liquid chromatography mass spectrometry as previously published (28). In subjects with type 2 diabetes and obesity, enrichments of $\left[6,6-{ }^{2} \mathrm{H}_{2}\right]$ glucose and ${ }^{2} \mathrm{H}_{5}$-glycerol were measured using gas chromatograph mass spectrometry as previously published (25). Validation of the comparability of these techniques was performed in a separate group of subjects (our unpublished observations). The turnover rate (rate of appearance) of glucose, glycer$\mathrm{ol}$, and palmitate was calculated by measuring isotope enrichment using the single pool model (29).

Fractional gluconeogenesis (i.e., the fraction of glucose derived from gluconeogenesis) was measured after the oral administration of deuterated water $\left({ }^{2} \mathrm{H}_{2} \mathrm{O}\right)$ as previously published (30). A total of $3 \mathrm{~g} / \mathrm{kg}$ lean body mass of ${ }^{2} \mathrm{H}_{2} \mathrm{O}$ was given in 3 or 4 divided doses between 9 $\mathrm{pm}$ and 3 am in order to enrich the subject's body water pool to approximately $0.3 \%{ }^{2} \mathrm{H}_{2} \mathrm{O}(25)$. Since body water serves as the precursor pool for deuterium, the average deuterium enrichment on glucose carbons $(\mathrm{C}-1,3,4,5,6,6)$ derived from body water during the gluconeogenic process is a measure of gluconeogenesis (29). Briefly, this method involves preparation of the pentaacetate derivative of glucose, followed by sample analyses using gas chromatography mass spectrometry in the positive chemical ionization mode (6890/5973, Agilent Technologies). Deuterium enrichment in plasma water was determined by isotope ratio mass spectrometry (Delta ${ }^{+} \mathrm{XL}$ IRMS, Thermo Finnigan) at Baylor College of Medicine for all subjects. The absolute rate of gluconeogenesis was calculated as the product of total glucose appearance rate and fractional gluconeogenesis. Glycogenolysis rate was calculated by subtracting the rate of gluconeogenesis from the glucose production rate.

Hepatic triglyceride content. Hepatic triglyceride content was measured by proton nuclear magnetic resonance spectroscopy (MRS) as previously described (31). In subjects with lipodystrophy, MRS was performed at the same visit as studies of gluconeogenesis and glycogenolysis. In subjects with INSR pathogenic variants, liver spectroscopy was performed 29 to 33 months after studies of gluconeogenesis and glycogenolysis; only 3 of the 7 subjects were available to have liver spectroscopy performed.

Body composition. Fat mass and fat-free mass were measured by dual energy x-ray absorptiometry as reported $(25,28,32)$.

Statistics. Statistical analyses were performed using GraphPad Prism version 8 (GraphPad Software) and SAS Enterprise Guide version 7.1. For continuous outcomes, normally distributed data are reported as the mean \pm SD and nonnormally distributed data are reported as the median (interquartile range). For comparisons of continuous outcomes between subjects with INSR pathogenic variants, lipodystrophy, type 2 diabetes, and obesity, mixed models were performed with post hoc pairwise comparisons and Tukey's correction for multiplicity. Sensitivity analyses were conducted for each model with the addition of age as a covariate and excluding patients with generalized lipodystrophy (Supplemental Table 2). Graphs highlighting data from patients with generalized lipodystrophy are shown in Supplemental Figures 1-3. Correlations among metabolic variables were assessed using Pearson correlations. Nonnormally distributed variables were log-transformed before analysis. $P$ values of less than 0.05 were considered statistically significant. All statistical tests were 2-sided. No sample size calculations were performed.

Study approval. Studies in subjects with lipodystrophy (NCT01778556, NCT00001987) and INSR pathogenic variants (NCT02457897) were approved by the NIH Institutional Review Board. Studies in subjects with type 2 diabetes and obese adolescent subjects without diabetes were approved by the Baylor College of Medicine Institutional Review Board. All subjects or their legal guardians provided written consent, and minors provided written assent, before inclusion.

\section{Author contributions}

HS analyzed data and wrote the manuscript. STC contributed to study design, conducted experiments, analyzed data, and contributed to manuscript writing. SC acquired and analyzed data and critically reviewed the manuscript. MWH contributed to study design and critically reviewed the manuscript. MS, MW, and PJW conducted experiments, acquired data, and critically reviewed the manuscript. ML analyzed data and critically reviewed the manuscript. RJB designed the study, conducted experiments, acquired data, analyzed data, and wrote the manuscript.

\section{Acknowledgments}

This work was supported by the intramural research programs of the National Institute of Diabetes and Digestive and Kidney Diseases (STC, MS, MW, PW, RJB) and the Eunice Kennedy Shriver National Institute of Child Health and Human Development (HS, ML). This work was also supported in part by federal funds from the USDA, Agricultural Research Service under Cooperative Agreement Number 58-3092-5-001 (to SC and MWH). We thank the volunteers (and their families) whose participation made this study possible. We gratefully acknowledge Elaine Cochran (NIDDK, Bethesda, Maryland, USA) and Susan Sharma (Department of Pediatrics, Baylor College of Medicine, Houston, Texas, USA) who facilitated patient care, data acquisition, and study execution. The contents of this publication do not necessarily reflect the views or 
policies of the US Department of Agriculture or the Department of Health and Human Services, nor does mention of trade names, commercial products, or organizations imply endorsement from the US government.

Address correspondence to: Rebecca J. Brown, National Institute of Diabetes and Digestive and Kidney Diseases, NIH, Building 10-CRC,
Room 6-5942, 10 Center Drive, Bethesda, Maryland 20892, USA. Phone:301.594.0609; Email: brownrebecca@mail.nih.gov.

HS's present affiliation is: Pediatric Obesity Program, Department of Pediatrics, College of Medicine, University of Tennessee Health Science Center and Children's Foundation Research Institute, Le Bonheur Children's Hospital, Memphis, Tennessee, USA.
1. Petersen MC, Shulman GI. Mechanisms of insulin action and insulin resistance. Physiol Rev. 2018;98(4):2133-2223.

2. Brown MS, Goldstein JL. Selective versus total insulin resistance: a pathogenic paradox. Cell Metab. 2008;7(2):95-96.

3. Shimomura I, Matsuda M, Hammer RE, Bashmakov Y, Brown MS, Goldstein JL. Decreased IRS-2 and increased SREBP-1c lead to mixed insulin resistance and sensitivity in livers of lipodystrophic and ob/ob mice. Mol Cell. 2000;6(1):77-86.

4. Musso C, et al. Clinical course of genetic diseases of the insulin receptor (type A and Rabson-Mendenhall syndromes): a 30-year prospective. Medicine (Baltimore). 2004;83(4):209-222.

5. Donnelly KL, Smith CI, Schwarzenberg SJ, Jessurun J, Boldt MD, Parks EJ. Sources of fatty acids stored in liver and secreted via lipoproteins in patients with nonalcoholic fatty liver disease. JClin Invest. 2005;115(5):1343-1351.

6. Vatner DF, et al. Insulin-independent regulation of hepatic triglyceride synthesis by fatty acids. Proc Natl Acad Sci USA. 2015;112(4):1143-1148.

7. Nakae J, Kitamura T, Silver DL, Accili D. The forkhead transcription factor Foxo1 (Fkhr) confers insulin sensitivity onto glucose-6-phosphatase expression. JClin Invest. 2001;108(9):1359-1367.

8. Puigserver $P$, et al. Insulin-regulated hepatic gluconeogenesis through FOXO1-PGC-1alpha interaction. Nature. 2003;423(6939):550-555.

9. O-Sullivan I, et al. FoxO1 integrates direct and indirect effects of insulin on hepatic glucose production and glucose utilization. Nat Commun. 2015;6:7079.

10. Perry RJ, et al. Hepatic acetyl CoA links adipose tissue inflammation to hepatic insulin resistance and type 2 diabetes. Cell. 2015;160(4):745-758.

11. Lu M, et al. Insulin regulates liver metabolism in vivo in the absence of hepatic Akt and Foxo1. Nat Med. 2012;18(3):388-395.
12. Titchenell PM, et al. Direct hepatocyte insulin signaling is required for lipogenesis but is dispensable for the suppression of glucose production. Cell Metab. 2016;23(6):1154-1166.

13. Petersen KF, et al. Leptin reverses insulin resistance and hepatic steatosis in patients with severe lipodystrophy. J Clin Invest. 2002;109(10):1345-1350.

14. Semple RK, Savage DB, Cochran EK, Gorden P, O'Rahilly S. Genetic syndromes of severe insulin resistance. Endocr Rev. 2011;32(4):498-514.

15. Safar Zadeh E, et al. The liver diseases of lipodystrophy: the long-term effect of leptin treatment. J Hepatol. 2013;59(1):131-137.

16. Stears A, O'Rahilly S, Semple RK, Savage DB. Metabolic insights from extreme human insulin resistance phenotypes. Best Pract Res Clin Endocrinol Metab. 2012;26(2):145-157.

17. Semple RK, et al. Postreceptor insulin resistance contributes to human dyslipidemia and hepatic steatosis. J Clin Invest. 2009;119(2):315-322.

18. Kawano Y, Cohen DE. Mechanisms of hepatic triglyceride accumulation in non-alcoholic fatty liver disease. J Gastroenterol. 2013;48(4):434-441.

19. Schwarz JM, Linfoot P, Dare D, Aghajanian K. Hepatic de novo lipogenesis in normoinsulinemic and hyperinsulinemic subjects consuming high-fat, low-carbohydrate and low-fat, high-carbohydrate isoenergetic diets. Am J Clin Nutr. 2003;77(1):43-50.

20. Diraison F, Moulin P, Beylot M. Contribution of hepatic de novo lipogenesis and reesterification of plasma non esterified fatty acids to plasma triglyceride synthesis during non-alcoholic fatty liver disease. Diabetes Metab. 2003;29(5):478-485.

21. Leavens KF, Birnbaum MJ. Insulin signaling to hepatic lipid metabolism in health and disease. Crit Rev Biochem Mol Biol. 2011;46(3):200-215.

22. Haas JT, et al. Hepatic insulin signaling is required for obesity-dependent expression of
SREBP-1c mRNA but not for feeding-dependent expression. Cell Metab. 2012;15(6):873-884.

23. Muoio DM, Newgard CB. Fatty acid oxidation and insulin action: when less is more. Diabetes. 2008;57(6):1455-1456.

24. McGarry JD. Banting lecture 2001: dysregulation of fatty acid metabolism in the etiology of type 2 diabetes. Diabetes. 2002;51(1):7-18.

25. Chung ST, Hsia DS, Chacko SK, Rodriguez LM, Haymond MW. Increased gluconeogenesis in youth with newly diagnosed type 2 diabetes. Diabetologia. 2015;58(3):596-603.

26. American Diabetes Association. Standards of medical care in diabetes--2011. Diabetes Care. 2011;34 Suppl 1:S11-S61.

27. Kinzer AB, Shamburek RD, Lightbourne M, Muniyappa R, Brown RJ. Advanced lipoprotein analysis shows atherogenic lipid profile that improves after metreleptin in patients with lipodystrophy. JEndocr Soc. 2019;3(8):1503-1517.

28. Brown RJ, et al. Metreleptin-mediated improvements in insulin sensitivity are independent of food intake in humans with lipodystrophy. J Clin Invest. 2018;128(8):3504-3516.

29. Wolfe R, Chinkes DL. Isotopic Tracers in Metabolic Research: Principles and Practice of Kinetic Analysis. Hoboken, New Jersey, USA: John Wiley \& Sons; 2005.

30. Chacko SK, Sunehag AL, Sharma S, Sauer PJ, Haymond MW. Measurement of gluconeogenesis using glucose fragments and mass spectrometry after ingestion of deuterium oxide. J Appl Physiol. 2008;104(4):944-951.

31. Muniyappa R, et al. Myocardial fat accumulation is independent of measures of insulin sensitivity. J Clin Endocrinol Metab. 2015;100(8):3060-3068.

32. Kushchayeva YS, et al. Thyroid hormone effects on glucose disposal in patients with insulin receptor mutations. JClin Endocrinol Metab. 2020;105(3):dgz079. 